Psychotherapeut 2022 67:129-134 https://doi.org/10.1007/s00278-021-00563-w Angenommen: 25. November 2021 Online publiziert: 10. Januar 2022

(c) Der/die Autor(en) 2021

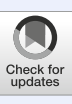

\section{Sprechen und Schweigen in der Psychotherapie}

\author{
Unterwegs zu einem kommunikativen "turn" \\ Michael B. Buchholz $\cdot$ Marie-Luise Alder ${ }^{2}$ (D) F Florian Dreyer $^{3}$ (D) $\cdot$ \\ Michael M. Franzen ${ }^{4}$ iD \\ 'International Psychoanalytic University (IPU) Berlin, Berlin, Deutschland \\ ${ }^{2}$ Arbeitsgemeinschaft für Psychoanalyse und Psychotherapie Berlin e. V. (apb), Berlin, Deutschland \\ ${ }^{3}$ Romanisches Seminar, Albert-Ludwigs-Universität Freiburg, Freiburg i. B., Deutschland \\ ${ }^{4}$ Arbeitsbereich Sozialpsychologie, International Psychoanalytic University (IPU), Berlin, Deutschland
}

Zusammenfassung

\section{In diesem Beitrag}

- Einführung

- Kurzer Rückblick auf psychoanalytische Positionen

- Konversationsanalyse Erzählen • Schweigen

- Folgerungen für die Praxis

Das Gespräch hat in jeder Psychotherapie eine zentrale Stellung. „Gesprächstherapie“ ist deshalb irreführend für eine besondere Art der Therapeutik. Im Folgenden wird von Analytiker(in) gesprochen, wenn diese Tradition gemeint ist, ansonsten von Therapeut(en) und Therapeutin. Probleme der Vertraulichkeit bei der Erforschung von Gesprächen können gut und sicher gelöst werden. Die Erforschung der therapeutischen Konversation ist weit vorangetrieben, von psychoanalytischer Seite und anderen weltweit. Da so wichtige Gesprächsmomente wie Schweigen und Erzählen vertieft verstanden werden konnten, wird ein „communicative turn“ vorgeschlagen: nicht

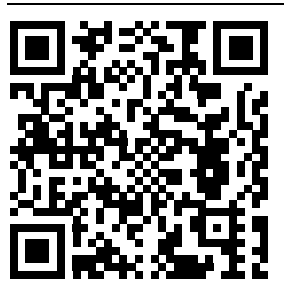

QR-Code scannen \& Beitrag online lesen länger eine jeweilige Psychotherapietheorie als Ausgangspunkt einer Untersuchung zu wählen, sondern das Gespräch selbst. Dazu werden Forschungsergebnisse mit Bezug zur klinischen Arbeit vorgestellt. Sie sind klinisch gehaltvoll.

\title{
Schlüsselwörter
}

Kommunikation · Konversationsanalyse · Erzählen · Psychoanalytische Deutung · BehandlerPatient-Beziehungen

Das Gespräch hat in jeder Psychotherapie eine zentrale Stellung. Da so wichtige Gesprächsmomente wie Schweigen und Erzählen inzwischen vertieft verstanden werden konnten, wird im Folgenden ein "communicative turn" vorgeschlagen: Es sollte nicht länger eine jeweilige Psychotherapietheorie als Ausgangspunkt einer Untersuchung gewählt werden, sondern das therapeutische Gespräch selbst.

\section{Einführung}

Am 07.02.2020 wurde der Helmut-ThomäGedächtnis-Preis an Jörg Bergmann verliehen. Helmut Thomä war neben Horst Kächele einer der Initiatoren in UIm, die sich mit dem sprachlichen Geschehen in therapeutischen Sitzungen seit dem Ende der 1960er-Jahre intensiv auseinanderzusetzen begannen. Bergmann ist Sozial- und Sprachwissenschaftler, der sich für jene sprachlichen Mittel, mit denen Psychotherapie betrieben wird, interessierte. $\mathrm{Er}$ machte auf eine Studie aufmerksam, die die ersten fünf Minuten eines Arzt-PatientGespräches untersuchte (Pittenger et al. 1960):

Ein Psychiater exploriert eine junge Frau. Er stellt ihr anamnestische Fragen, die sie knapp beantwortet. Bei der Frage nach ihrem Beruf gibt sie an, Krankenschwester zu sein. Ihr Mann verbiete ihr, zu arbeiten. Darauf fragt der Arzt: „Wie alt sind Sie?" Die Patientin nennt ihr Alter, Mitte dreißig. Im „Schatten“ der anamnestischen Befragung stellt sich unerwartet eine zweite Bedeutung, ein verwunderter Kommentar zur fraglichen ehelichen Gefügigkeit, ein. Ob es Absicht war, wissen wir nicht. Es verdeutlicht, wie vieldeutig Fragen werden können. Unerwartete und nichtintentional herbeigeführte Be- 
deutungswechsel können zu kurativen Faktoren im Gespräch werden; jeder, der klinisch arbeitet, kennt ähnliche Situationen. $\mathrm{Zu}$ viele kommunikative Ereignisse fallen „unter den Tisch“.

Ein Arzt fragt einen Patienten, seit wann sein Leiden besteht. Seine Antwort: "Seit zwei Jahren" - und fügt leise nach kurzem Zögern an: "Seit dem Tod meiner Frau“. Ins Datenblatt aber trägt der Arzt nur den ersten Teil der Antwort ein (Kanwischer 2021). Das ist ",korrekt", die therapeutisch relevante zweite Antwort jedoch wird in den Registrierungstechniken "gelöscht". Die Schulung junger Therapeuten in der Anwendung von Fragebogen und Manualen mit dem Ziel einer Objektivierung, wird hier fraglich. Schließt man Prosodie, Blicke, Gestik und andere „körperliche" Momente der Konversation mit ein, wird das deutlich; und noch mehr, wenn man Schweigen dazu nimmt. Wie beredt Schweigen sein kann, weiß der Volksmund schon lange, man kann es detailreich untersuchen (Dimitrijević und Buchholz 2021). Dem „sprechenden Geschehen“ im Behandlungszimmer wird in der Prozessforschung seit längerer Zeit verstärkt Aufmerksamkeit gewidmet. Dazu gibt es exzellente Überblicke (Gumz et al. 2019; Marx et al. 2017). Die Autoren des vorliegenden Beitrags wollen anregen, dem in Ausbildung und Praxis weit mehr Aufmerksamkeit zu widmen.

\section{Kurzer Rückblick auf psycho- analytische Positionen}

Zentrales Element psychoanalytischer Behandlungsführung war zu Anfang nicht die „Deutung", sondern das Schweigen, „auch in der Analyse [sei] ... das Gesprochene als solches nicht das Wichtigste". Relevanter sei „zu erkennen, was das Sprechen verschweigt und das Schweigen spricht" (Reik 1928, S. 85). Je mehr sich „Relationalität" und Arbeitsbündnis in der Psychoanalyse durchzusetzen begannen, umso relevanter wurde die "horizontale" Dimension des Sozialen-im-Sprechen gegenüber einer „vertikalen“ Tiefendimension. Damit gewann Aufmerksamkeit, was als unbewusster Dialog (Ferenczi 1915) thematisiert worden war. Hier ergeben sich Kooperationen mit anderen Untersuchungsmethoden wie $z$. B. der Konversationsanalyse (KA; Buchholz und Kächele 2013).
Psychoanalytische Autoren (Krejci 2009; Levy und Inderbitzin 1990; Paniagua 1991; Smith 1993) begannen mit der Untersuchung der Gesprächsdetails; ein Ergebnis war die paradox klingende Aufforderung, sich „in die Oberfläche zu vertiefen“. In Deutschland waren starke Impulse zur Prozess- und Gesprächsanalyse schon sehr früh gestartet worden (Thomä und Kächele 2021 [1985]). Bezüge zu „Prinzipien“ therapeutischer Praxis rückten ins Aufmerksamkeitszentrum.

Ein Beispiel: Spence et al. (1994) zeigten am transkribierten Material einer mehr als 500 h dauernden Analyse, wie Patienten unbewusst ihren Analytiker steuern: Wenn sie ihn früh in der Stunde ansprechen (,Sie sagten gestern ..."), dann reden Analytiker nicht nur früher, sondern auch mehr, als wenn eine solche direkte Anrede fehlt. Diese Studie zeigte, dass Therapeuten keineswegs unabhängig „intervenieren“, sondern ihr Tun geradezu gesteuert ist von dem, was Patienten tun. Es hatte sich bislang der Selbstanalyse entzogen. Konzepte wie Übertragung und Gegenübertragung sind zu großformatig, um prozessrelevante Feinheiten, die sich aus dem Studium der Gesprächsdetails ergeben, zu erfassen.

\section{Konversationsanalyse}

Diese Entwicklung wird von Entwicklungen der Psychotherapieforschung flankiert. Drei ehemalige Herausgeber des führenden Journals Psychotherapy Research formulierten 2015 die Sorge, die Schwerpunktbildung auf dem "Outcome" sei ohne das, was im Prozess geschehe, sinnlos geworden. Alle Bona-fide-Therapien erbringen äquivalente Ergebnisse, doch die Theorien der therapeutischen Schulen stehen widersprüchlich zueinander. Dieses Äquivalenzparadox könne nur durch verstärkte Prozessforschung bearbeitet werden. Sie schlagen als Wege für zukünftige Forschung vor: Fallstudien, KA sowie umfangreiche und anspruchsvollere Prozessanalysen (Stiles et al. 2015, S. 288). Die KA wird hier neben andere Ansätze gestellt. Die „Oberfläche des Gesprächs" wird rehabilitiert, sie ist nicht oberflächlich. Sehr viel ist geleistet, was hier nur summarisch dargestellt werden kann. Konversationsanalyse und Psychotherapie lernen voneinander. Einige interessan- te Themen, die von der KA beobachtet wurden:

a) Patienten haben privilegierten $\mathrm{Zu}$ gang zu persönlichen Erfahrungen; deshalb müssen Therapeuten sich immer erst die Berechtigung erwerben, dem Patienten zu verdeutlichen, dass seine Erfahrung manchmal von dem abweichen kann, was er glaubt, was sie sei (Voutilainen und Peräkylä 2015). Therapeuten benutzen dazu „Formulierungen" (Dreyer und Goßmann 2014): Der Patient teilt mit, er sei „traurig", aber der Therapeut "formuliert", er sei „nicht nur traurig, sondern wütend, vielleicht sogar ziemlich wütend" (Rae 2008).

b) Manchmal sagen Therapeuten ihren Patienten, dass sie fühlen oder handeln wie ... eine Figur aus ihren Erzählungen. Diese empathische Leistung verknüpft verschiedene, räumlich oder zeitlich divergente Erfahrungen miteinander, und wenn das gelingt (Peräkylä 2019), verschiebt sich deren konzeptuelle Rahmung: Dasselbe Erleben wird in einem anderen Bezugsrahmen thematisierbar. Und mehr noch: Der Patient wird sich bewusst, dass er nicht nur unter gegenwärtigen Umständen leidet, sondern die Gegenwärtigkeit solcher Umstände schon immer in diesem Bezugsrahmen unbewusst annimmt (Ekberg und LeCouteur 2015).

c) Im deutschen Sprachraum war früh beobachtet worden, wie irritiert Patienten auf kommunikative Eigenarten reagieren, etwa, wenn sie auf Fragen keine bzw. nur verspätete Antworten bekommen (Flader 1991). Sie brauchen dazu Erläuterungen und Begründungen.

d) Therapeuten sind nicht die Einzigen, die "deuten", sondern Patienten nehmen höchst subtil Widersprüche oder Irritationen ihres Therapeuten wahr (Alder 2020); sie müssen ermutigt werden, das ihrerseits mitzuteilen. Das wird in der KA als kommunikativ ausgehandelte Berechtigungen thematisiert („Deontik“; Stevanovic und Peräkylä 2012). Hier wird eine ethisch relevante Dimension bearbeitet (Stevanovic 2018).

Das hat Folgen: Auch in anderen Traditionen (J. Habermas 1981) ist bekannt, dass schon in jedem alltäglichen Gespräch das, was ein anderer sagt, nicht einfach als "Information" aufgenommen wird, sondern Interpretation beständig im Spiel ist. Darin zeigen sich die Menschen als kultu- 
relle Wesen: „Kultur nenne ich den Wissensvorrat, aus dem sich die Kommunikationsteilnehmer, indem sie sich über etwas in einer Welt verständigen, mit Interpretationen versorgen" (J. Habermas 1981, Bd. 1, S. 209). Die Unverzichtbarkeit kultureller Reserven für die Deutung in der Sitzung wurde durch KA-Studien belegt (Scarvaglieri 2020). Deutungen stellen Zusammenhänge zwischen Erfahrungsbereichen her (Peräkylä 2004, S. 293).

Für die Praxis bedeutet das: a) Therapeuten erwerben die "Lizenz zur Deutung" nicht durch Ausbildung, sondern müssen vom Patienten individuell autorisiert werden. b) Diese "Lizenz" muss von anderen Mitteilungen unterschieden werden. c) Sie deuten weniger Kausalität von Ereignissen, sondern helfen, die Verarbeitung einer Erfahrung („framing ") als Teil des Problems bzw. des Konflikts in den Blick zu nehmen. d) Das mentale Blickfeld des Patienten erweitert sich so; der Patient gewinnt eine "exzentrische" Position. Wenn er eine Erfahrung eben noch im „alten“, dann aber im "neuen" Rahmen miteinander vergleicht, tut er das unvermeidlich aus einer Position, die weder der alte noch der neue Rahmen ist. Und dennoch weiß er, dass er eben noch „innerhalb“ des einen Rahmens festgesteckt hatte.

Das konnte die KA aus transkribierten psychoanalytischen Sitzungen gut ermitteln (Peräkylä 2005): Der Patient erzählt, der Therapeut macht einen Deutungsvorschlag, der Patient korrigiert die Formulierung, und der Therapeut akzeptiert diese Korrektur. Vier Schritte also, statt nur einer. Keineswegs ist der Analytiker überlegener Alleswisser. Er muss sich, besonders im vierten dieser Schritte korrigieren lassen können - warum? Weil sonst das interaktive Gleichgewicht der Kooperation gefährdet wäre. Die Deutung enthält unvermeidlich einen kritischen Anteil. Gelingt ein solcher Schritt, kommt es zur Vertiefung der Beziehung. Korrekturen eröffnen interaktionale Räume, in denen unter dem scheinbar neutralen Vorwand der Korrektur auch ganz andere schwierigere Themen verhandelt werden können (Bergmann und Drew 2017).

Die Annahme, dass eigene Erlebnisse nicht alles sind, sondern dass entscheidend wird, als was etwas erlebt wird, hat nicht nur dem Wörtchen „als“ besonderen
Platz in der Professionstheorie der Psychotherapie eingeräumt (Buchholz 1999). Die KA hat noch Weiteres beigesteuert. Ein Überblick über jüngste konversationsanalytische Forschungen zu "Talking and cure" findet sich in dem gleichnamigen „research topic“ (Buchholz und Peräkylä 2021).

\section{Erzählen}

In der Narrationsforschung (T. Habermas 2018; T. Habermas und Berger 2011) war man lange Zeit davon ausgegangen, dass der Erzähler spricht und der Zuhörer hört, also an der Erzählung nicht beteiligt sei. Tatsächlich werden Erzählungen (Scheidt et al. 2015) am Zuhörer orientiert. Von deren Aufmerksamkeit überzeugen sich Sprecher mithilfe von Blicken, die immer wieder rasch auf Zuhörer gerichtet werden. Sprecher hören, ob Zuhörer sich auf ihre Seite schlagen, ob die Erzählung ihren "state" (Zustand) verändert hat. Dazugehörige Äußerungen heißen "changeof-state token" (CoST; Heritage 1984) und können durch verwundertes Kopfschütteln oder Ausrufe wie "wow!" oder dergleichen eingebracht werden. Wenn der COST ausbleibt, der Zuhörer die Stellungnahme oder den Blick vermeidet, wiederholt der Sprecher seine Geschichte mehrfach, um die Stellungnahme doch noch zu elizitieren.

Warum ist das so? Das hat eine Studie (Peräkylä et al. 2015) ermitteln können. Interaktanten wurden „verdrahtet“; während des Erzählens und Zuhörens wurden Herzrate, psychogalvanische Hautreaktion u. a. physiologische Maße gemessen. Dabei zeigt sich, dass die in der qualitativen Narrationsforschung beobachtete Spannungssteigerung sich auch physiologisch dokumentiert. Neuartig ist die Beobachtung, dass die physiologische Spannung des Erzählers schlagartig abfällt, wenn der Hörer ein COST vernehmen lässt und weiter, dass die physiologische Spannung des Zuhörers sich dabei bis auf die Höhe des Sprechers steigert. Das nennen die Autoren "sharing the emotional load". Tatsächlich ist hier ein Beleg dafür erbracht, wie Therapeuten emotionale Last mit ihren Patienten teilen. Dieser Befund konnte bestätigt werden (Voutilainen et al. 2018).
Erzählungen ist gemeinsam, dass Erzähler Anteilnahme und Solidarität suchen. Die Narrationsforschung spricht vom „performativen Selbst", das die Kongruenz der Relevanzsysteme wiederherstellen möchte, v. a. aber die Austauschbarkeit der Perspektiven. Es kann sich „beruhigen“, wenn diese "geliefert" wurde (Deppermann et al. 2020). Zuhörer allerdings achten meist auf das "erzählte Selbst", also auf die Figur innerhalb der Erzählung und verfolgen den Inhalt. Therapeuten können lernen, dass man gegenüber dem performativen Selbst freilich nicht neutral bleiben kann. Eine Erzählung nur anzuhören, ist nicht neutral, sondern verweigert die geforderte Stellungnahme. Eine Erzählung, dass man vom Chef als "Trottel" bezeichnet wurde, ohne verwunderte Reaktion zu lassen, bestätigt unvermeidlich den Chef, ist also keineswegs neutral. „Neutralität" kann verletzen.

Gelingt die interaktive Erzählung, dann können Therapeuten die Aufmerksamkeit ihrer Patienten vom „erzählten“ zum „performativen" Selbst lenken; sie gehen zunächst auf Gefühle und Inhalte einer Erzählung ein, dann auf die Art des Erzählens (Performanz) über, weil nur hier vertiefte therapeutische Einsichten zu erreichen sind.

\section{Schweigen}

In der Psychotherapieforschung war dem Schweigen, anders als in Kulturwissenschaft oder Philosophie, nur geringe Aufmerksamkeit gewidmet worden. Levitt entwickelte ein System, womit Pausen klassifiziert werden konnten - je danach, wie es weiterging. Das System unterschied in 7 Stufen obstruktive von kreativen Pausen, und dann zeigte sich, dass der Behandlungserfolg statistisch aus der Häufigkeit des Pausentyps vorhergesagt werden konnte: Therapien mit gehäuften obstruktiven Pausen hatten weniger, die mit kreativen Pausen besseren Erfolg (Levitt und Morrill 2021).

Eine andere Studie (Dreyer und Franzen 2021) untersuchte Pausen in therapeutischen Sitzungen von mehr als 30 s Dauer. Sie beobachtete in der Auswertung von 45 transkribierten Sitzungen aus dem Projekt Conversation Analysis of Empathy in Psychotherapy Process Research (CEMPP; 
durchgeführt an der IPU) folgendes Muster: a) Patienten berichten $\left(R={ }_{\text {}}\right.$ report $\left.{ }^{\prime \prime}\right)$ ein persönliches Problem. b) Es kommt zu einem längeren Schweigen ( $L=$ "lapse“). c) Anschließend bezieht sich ( $R=$ "refers") die Patientin auf ihre gedanklichen Prozesse während des Schweigens. d) Beide untersuchen diese, um eine gemeinsame Vorstellung ( $I=$, image $\left.{ }^{\prime \prime}\right)$ davon zu entwickeln und zu prozessieren. Das RLRI-Modell ergab verschiedene Beobachtungen, von denen eine hier genannt werden soll: Patienten kehren nach dem Schweigen zu ihnen allein zugänglichen Prozessen gedanklicher Art zurück. Das entstehende epistemische Ungleichgewicht - der Therapeut kann davon nichts wissen - steuert die weitere Ausarbeitung eines Themas und mündet in ein gemeinsames, bildhaftmetaphorisches Thema. Daran können beide kommunikativ teilhaben, und so wird das Ungleichgewicht in einer gemeinsamen Bildkonstruktion aufgelöst. Diese ist in der Interaktion kokonstruiert, verhandelbar und sichert die gemeinsame Basis, dass beide sich affektiv, imaginativ und kommunikativ noch "in derselben Welt" befinden.

Eine weitere Studie (Buchholz et al. 2021) handelt von einem Schweigen, das nach dem Wissen der Autoren des vorliegenden Beitrags bisher noch nie untersucht wurde. Es konnte bei einem videografierten Paargespräch deutlich gemacht werden, dass, während die beiden sprachen, etwas verschwiegen wurde. Es gibt ein Schweigen im Sprechen. Jeder Sprecher äußert unvermeidlich eine Erwartung an den Hörer, der seine Äußerung deshalb „im Rahmen des Erwartbaren“ abgibt. Ist das nicht der Fall, bleibt etwas in der Luft hängen. Im untersuchten Paargespräch war das die Situation, dass beide einander aufforderten, zu bedeutsamen Themen Stellung zu nehmen. Und antworteten, indem zitiert wurde, was Mutter oder ein Freund dazu meinten - im zitierenden Sprechen anderer dokumentierte sich das eigene Schweigen. „Hearing silencing ", Vernehmen des Verstummten, bleibt eine immerwährende praktische Aufgabe, in der Therapeuten geschult werden können.

\section{Folgerungen für die Praxis}

a) Was in der Psychotherapie hilft, ist das Gespräch. Ein genauerer Blick für die Art und Weise, wie Therapeuten mit Patienten sprechen und schweigen, sollte in die Ausund Weiterbildung aufgenommen werden. Freilich - nahezu alle Therapeutiken haben keine umfängliche Aufmerksamkeit für das eigene Sprechen entwickelt, was erstaunlich genug ist.

b) Wenn mal etwas "nicht so gut" läuft, sollte der Blick nicht nur in die (biografische) Tiefe, sondern in die erlebte Aktualität im Behandlungszimmer gerichtet werden.

c) Die Verallgemeinerung von Befunden gelingt, etwa in der Studie von Spence et al. (s. Abschn. „Kurzer Rückblick auf psychoanalytische Positionen"). Jedoch - menschliche Gespräche sind so facettenreich, dass man eher etwas dazu sagen kann, was im Gespräch nicht stattfinden sollte. Ein Beispiel wäre jener Therapeut, der jede seiner (ansonsten gehaltvollen) Äußerungen mit „Ja - aber ..." einleitete und damit einen Kontrast aufbaute, der die Patientin provozieren musste. Ein anderes Beispiel wurde unter "Neutralität" genannt. Tatsächlich informiert die Forschung, dass gute Therapeuten für jeden Patienten eine individuelle Therapie „erfinden“ (Norcross und Wampold 2018). Die Suche nach allgemeinen Regeln gelingt eher rekonstruktiv im Nachhinein, kaum aber als Vorschrift oder Direktive. Aber sich damit zu beschäftigen, schult die Aufmerksamkeit sehr wohl.

d) Die großen Metaanalysen haben gezeigt, dass Psychotherapie hilft, aber nicht „wie“. Die übermächtige Diskussion der Theorie der jeweiligen therapeutischen „Schulen“ übersieht oft die Relevanz und Bedeutung des unmittelbaren Interaktionsgeschehens.

e) Warnend hatte Wampold (2001, S. 2, Übersetzung M.B.B.) in seiner Zusammenschau aller relevanten Studien formuliert: „... dass die Psychotherapie mit dem medizinischen Modell unvereinbar ist, und dass die Konzeptualisierung der Psychotherapie auf diese Weise die therapeutische Anstrengung verzerrt. Dringlicher ausgedrückt: Die Medikalisierung der Psychotherapie könnte die Psychotherapie als nützli- che Behandlung psychologischer und sozialer Probleme zerstören."

\section{Fazit für die Praxis}

- Konversation („,Gespräch“) ist ein zutiefst menschliches Werkzeug, dessen wohltuende, ja heilende Wirkung die Menschen gerade in der Coronapandemie vermissen.

- Die Analyse der Konversation hat das Potenzial, die Details therapeutischen Sprechens in ihrer immensen Bedeutung hervortreten zu lassen.

- Ein „kommunikativer turn“ ist überfällig, d.h. nicht länger eine jeweilige Psychotherapietheorie als Ausgangspunkt einer Untersuchung zu wählen, sondern das Gespräch selbst.

\section{Korrespondenzadresse}

Prof. Dr. Michael B. Buchholz

International Psychoanalytic University (IPU)

Berlin

Stromstr. 3, 10555 Berlin, Deutschland

michael.buchholz@ipu-berlin.de

Danksagung. Ein herzlicher Dank an Frau Prof. Dr. Dorothea Huber für die zur Verfügung gestellten Audioaufnahmen aus der Münchner Psychotherapiestudie.

Funding. Open Access funding enabled and organized by Projekt DEAL.

\section{Einhaltung ethischer Richtlinien}

Interessenkonflikt. M.B. Buchholz, M.-L. Alder, F. Dreyer und M.M. Franzen geben an, dass kein Interessenkonflikt besteht.

Für diesen Beitrag wurden von den Autoren keine Studien an Menschen oder Tieren durchgeführt. Für die aufgeführten Studien gelten die jeweils dort angegebenen ethischen Richtlinien.

Open Access. Dieser Artikel wird unter der Creative Commons Namensnennung 4.0 International Lizenz veröffentlicht, welche die Nutzung, Vervielfältigung, Bearbeitung, Verbreitung und Wiedergabe in jeglichem Medium und Format erlaubt, sofern Sie den/die ursprünglichen Autor(en) und die Quelle ordnungsgemäß nennen, einen Link zur Creative Commons Lizenz beifügen und angeben, ob Änderungen vorgenommen wurden.

Die in diesem Artikel enthaltenen Bilder und sonstiges Drittmaterial unterliegen ebenfalls der genannten Creative Commons Lizenz, sofern sich aus der Abbildungslegende nichts anderes ergibt. Sofern das betreffende Material nicht unter der genannten Creative Commons Lizenz steht und die betreffende Handlung nicht nach gesetzlichen Vorschriften erlaubt ist, ist für die oben aufgeführten Weiterverwendungen des Ma- 
terials die Einwilligung des jeweiligen Rechteinhabers einzuholen.

Weitere Details zur Lizenz entnehmen Sie bitte der Lizenzinformation auf http://creativecommons.org/ licenses/by/4.0/deed.de.

\section{Literatur}

Alder M-L (2020) Allusives Sprechen in Psychotherapien. Humboldt-Universität zu Berlin, Berlin. https://doi.org/10.18452/21288

Bergmann JR, Drew P (2017) Introduction. In: Jefferson $G$ (Hrsg) Repairing the broken surface of talk. Managing problems in speaking, hearing, and understanding in conversation. Oxford University Press, New York, S 1-26 (ed. by Jörg R. Bergmann \& Paul Drew)

Buchholz MB (1999) Psychotherapie als Profession. Psychosozial, Giessen

Buchholz MB, Kächele H (2013) Conversation analysis-A powerful tool for psychoanalytic practice and psychotherapy research. Lang Psychoanal 2(2):4-30

Buchholz MB, Ehmer O, Mahlstedt C, Pfänder $S$, Schumann E (2021) Speaking that silences. A single case multi-method analysis of a couple's interview. In: Dimitrijević A, Buchholz MB (Hrsg) Silence and silencing in psychoanalysis. Cultural, clinical, and research aspects. Routledge, New York, S333-397

Buchholz MB, Peräkylä A (Hrsg) (2021) Research topic: "talking and cure" - what's really going on in psychotherapy". https://www.frontiersin.org/ research-topics/11485/talking-and-cure--whats-really-going-on-in-psychotherapy\# articles.Zugegriffen: 19. Sept. 2021

Deppermann A, Scheidt CE, Stukenbrock A (2020) Positioning shifts. From told self to Performative self in psychotherapy. Front Psychol. https://doi. org/10.3389/fpsyg.2020.572436

Dimitrijević A, Buchholz MB (Hrsg) (2021) Silence and silencing in psychoanalysis. Cultural, clinical, and research aspects. Routledge, New York

Dreyer F, Franzen MM (2021) How to move on after silences. Adressing thought processes to restart conversation. In: Dimitrijević A, Buchholz MB (Hrsg) Silence and silencing in psychoanalysis. Cultural, clinical, and research aspects. Routledge, New York, S275-306

Dreyer F, Goßmann M (2014) Die „Formulierung" als therapeutisches Referenzieren. Selbstpsychologie 15(51):80-104

Ekberg K, LeCouteur A (2015) Clients' resistance to therapists' proposals. Managing epistemic and deontic status. J Pragmat 90:12-25. https://doi. org/10.1016/j.pragma.2015.10.004

Ferenczi S (Hrsg) (1915) Theorie. Bausteine zur Psychoanalyse, Bd. Band I. Ullstein, Frankfurt, Berlin, Wien (1984)

Flader D (Hrsg) (1991) Verbale Interaktion - Studien zur Empirie und Methodologie der Pragmatik. Metzler, Stuttgart

Gumz A, Reuter L, Flückiger C, Marx C, Rugenstein K, Schlipfenbacher $C$ et al (2019) Umgang mit Spannungen und Krisen in der therapeutischen Beziehung: Erste Erfahrungen mit einem handlungsorientierten Ausbildungs- und Supervisionskonzept. Psychother Psychosom Med Psychol. https://doi.org/10.1055/a-0892-3886 (Advance online publication)

Habermas J (1981) Theorie des kommunikativen Handelns. Suhrkamp, Frankfurt/M. (2 Bde)

\section{Speaking and silence in psychotherapy. On the way to a communicative turn}

Talk in interaction, conversation, has a central position in every psychotherapy.

Therefore, "conversational therapy" is misleading for a particular form of therapy. In this article the term analyst is used when this tradition is meant, otherwise the term therapist is used. Problems of confidentiality in the exploration of conversations can be easily and safely resolved. The exploration of therapeutic conversation has been widely advanced by psychoanalytic researchers and others worldwide. As important moments of conversation, such as silence and narration, could be understood more deeply, a communicative turn is proposed: a particular theory of psychotherapy is no longer selected as the starting point of an investigation but the conversation itself. To this end, research findings related to clinical work are presented here. They are clinically substantial.

\section{Keywords}

Communication · Conversation analysis · Narration · Psychoanalytical interpretation · Professionalpatient relations

Habermas T (2018) Emotion and narrative Bd. 1-3. Cambridge University Press, New York https:// doi.org/10.1017/9781139424615

Habermas T, Berger N (2011) Retelling everyday emotional events: Condensation, distancing, and closure. Cogn Emot 25(2):206-219. https:// doi.org/10.1080/02699931003783568

Heritage J (1984) A change-of-state token and aspects of its sequential placement. In: Atkinson JM, Heritage J (Hrsg) Structures of social action. Cambridge University Press, New York, $\mathrm{S}$ 299-346

Kanwischer H (2021) Wenn das Leben zum Überleben verkleinert wird - Über Risikoeinschätzung in der Dynamik von Angsterkrankungen. 1bis19 Initiative für Grundrechte und Rechtsstaat e. V. Berlin

Krejci E (2009) Immersion in the surface. Int J Psychoanal 90:827-842

Levitt HM, Morrill Z (2021) Measuring silence: The pausing inventory categorization system and a review of findings. In: Dimitrijević $A$, Buchholz MB (Hrsg) Silence and silencing in psychoanalysis. Cultural, clinical, and research aspects. Routledge, New York, S233-250

Levy ST, Inderbitzin LB (1990) The analytic surface and the theory of technique. J Am Psychoanal Assoc 38:371-391

Marx C, Benecke C, Gumz A (2017) Talking cure models - A framework of analysis. Front Psychol 8:367. https://doi.org/10.3389/fpsyg.2017. 01589

Norcross JC, Wampold BE (2018) Anew therapy for each patient: Evidence-based relationships and responsiveness. J Clin Psychol 74(11):1889-1906. https://doi.org/10.1002/jclp.22678

Paniagua C (1991) Patient's surface, clinical surface, and workable surface. J Am Psychoanal Assoc 39:669-685

Peräkylä A (2004) Making links in psychoanalytic interpretations: a conversation analytical perspective. Psychother Res 14:289-307

Peräkylä A (2005) Patients' responses to interpretations: A dialogue between conversation analysis and psychoanalytic theory. Commun Med 2:163-176

Peräkylä A (2019) Conversation analysis and psychotherapy: identifying transformative sequences.
Res Lang Soc Interact 52(3):257-280. https://doi. org/10.1080/08351813.2019.1631044

Peräkylä A, Henttonen P, Voutilainen L, Kahri M, Stevanovic M, Sams M, Ravaja N (2015) Sharing the emotional load. Recipient affiliation calms down the storyteller. Soc Psychol Q 78(4):301-323. https://doi.org/10.1177/0190272515611054

Pittenger RE, Hockett CF, Danehy JJ (1960) The first five minutes. A sample of microscopic interview analysis. Paul Martineau, New York

Rae J (2008) Lexical substitution as a therapeutic resource. In: Peräkylä A, Antaki C, Vehviläinen S, Leudar I (Hrsg) Conversation analysis and psychotherapy. Cambridge University Press, Cambridge, New York, S62-79

Reik T (1928) Das Schweigen. In: Storfer AJ (Hrsg) Almanach der Psychoanalyse, Bd. 3. Internationaler psychoanalytischer Verlag, Wien, S72-85

Scarvaglieri C (2020) First encounters in psychotherapy: relationship building and the pursuit of institutional goals. Front Psychol 11(585038):1-14

Scheidt EC, Lucius-Hoene G, Stukenbrock A, Waller E (Hrsg) (2015) Narrative Bewältigung von Trauma und Verlust, 1. Aufl. Schattauer, Stuttgart

Smith HF (1993) The analytic surface and the discovery of enactment. Annu Psychoanal 21:243-255

Spence DP, Mayes LC, Dahl H (1994) Monitoring the analytic surface. J Am Psychoanal Assoc 42:43-64

Stevanovic M (2018) Social deontics: A nano-level approach to human power play. J Theory Soc Behav 13(4):361. https://doi.org/10.1111/jtsb. 12175

Stevanovic M, Peräkylä A (2012) Deontic authority in interaction: the right to announce, propose, and decide. Res Lang Soc Interact 45(3):297-321

Stiles WB, Hill CE, Elliott R (2015) Looking both ways. Psychother Res 25(3):282-293. https://doi.org/ 10.1080/10503307.2014.981681

Thomä H, Kächele H (2021) Practice, Revised Second Edition. Aufl. Psychoanalytic therapy—-principles and practice, Bd. Vol.2. Psychosozial, Giessen (1985)

Voutilainen L, Peräkylä A (2015) Therapeutic conversation. In: Östman J-O, Verschueren J (Hrsg) Handbook of pragmatics. John Benjamins, New York

Voutilainen L, Henttonen P, Kahri M, Ravaja N, Sams M, Peräkylä A (2018) Empathy, challenge, and 
psychophysiological activation in therapist-

client interaction. Front Psychol. https://doi.org/

10.3389/fpsyg.2018.00530

Wampold BE (2001) The great psychotherapy deba-

te-models, methods and findings. Lawrence

Earlbaum, Mahwah, London

Hier steht eine Anzeige.

Springer 